\title{
Nonlinear dynamics of a dense two-dimensional dipolar exciton
}

\author{
gas \\ Ronen Rapaport, Gang Chen, and Steven H. Simon ${ }^{1}$ \\ ${ }^{1}$ Bell Laboratories, Lucent Technologies, \\ 600 Mountain Avenue, Murray Hill, New Jersey 07974
}

\begin{abstract}
We use a simple model to describe the nonlinear dynamics of a dense two dimensional dipolar exciton gas. The model predicts an initial fast expansion due to dipole-dipole pressure, followed by a much slower diffusion. The model is in very good agreement with recent experimental results. We show that the dipole pressure induced expansion strongly constrains the time available for achieving and observing Bose-Einstein quantum statistical effects, indicating a need for spatial exciton traps. We also suggest that nonlinear ballistic exciton transport due to the strong internal dipole pressure is readily achievable.
\end{abstract}


A new species of exciton, the spatially indirect dipolar exciton, has recently emerged in the continuing search for degenerate quantum gases and liquids in excitonic systems [1, 2]. Spatially indirect dipolar excitons are coulomb bound electron-hole pairs in which the electron and the hole are confined in two spatially separated two-dimensional (2D) layers. Such excitonic states are usually achieved in double quantum well (DQW) structures with an external electrical bias applied perpendicular to the quantum well (QW) plane to lift the degeneracy of the confined states in the two respective QW's [1, 2]. As a result, the lowest energy optical transitions occur between the two QW's, leading to the formation of indirect excitons. While the binding energy of such an exciton is only slightly modified due to the spatial separation, the lifetime is enhanced by many orders of magnitude compared to direct excitons (where the electron and the hole are in the same layer). This extremely long lifetime makes them especially interesting in the context of degenerate quantum gases of excitons, since it may provide sufficient time for thermalization as well as cooling of initially hot excitons. Another prominent feature of this type of indirect exciton is its permanent dipole moment, aligned perpendicular to the QW plane, due to the spatial separation of the electron and the hole. This dipole moment gives rise to a repulsive dipole-dipole force between excitons. On one hand, this repulsion prevents the formation of e-h complexes such as biexcitons and e-h droplets, and therefore makes such systems even more attractive candidates for observing degenerate Bose-Einstein gases. On the other hand, it leads to a strong driven expansion of a dense dipolar exciton gas, as was very recently observed [3] $]$. Hence, this dipole-dipole repulsion seems to constrain the time that such an indirect exciton gas can remain dense after photoexcitation, an obvious obstacle to the observation of quantum statistical effects of excitons.

Here, we use a simple model to describe the dynamics of such dipolar excitons, taking into account the dipole-dipole interaction. Besides quantitatively describing current experimental observations of the nonlinear expansion dynamics of dipolar exciton gases, this model suggests that the expansion caused by the dipole repulsion indeed strongly limits the time that a high mobility exciton gas remains dense, explaining the difficulties in the observations of exciton condensation. Some sort of spatial confining (exciton traps) might then be necessary for achieving a dense and cold dipolar exciton gas, a prerequisite for excitonic Bose-Einstein condensation in such systems.

Two recent experimental observations of dipolar exciton dynamics by Voros et al. 
(Ref. 3]) are the basis of our model assumptions: (a) The dipolar exciton expansion dynamics can be divided into two regimes: immediately after the laser excitation pulse, a very fast expansion is observed, in which the excited exciton cloud expands from its initial small diameter $(\sim 30 \mu m)$ by a factor of $\sim 6$, in a few tens of nanoseconds. At later times, the expansion slows down significantly, and can be very well described by exciton diffusion process.

(b) The diffusion coefficient of the dipolar excitons, $D_{X}$, has a power law dependence on the well width, $L$, where it is found to be of the form $D_{X} \propto L^{6}$. This specific dependence was found previously for free electrons in similar structures by Sakaki et al. in Ref. 4], which is well explained by interface roughness scattering from well width fluctuations [5, 6, 7]. This finding is a strong evidence that for dipolar excitons at low temperatures, potential fluctuations due to interface roughness is also the dominant scattering mechanism.

Based on these two observations, we suggest a simple model to describe the dynamics of the dipolar excitons (see also Ref. [8]). The model includes the dipole-dipole repulsion between the excitons as the main driving force for the initial fast expansion, and the static interface roughness scattering as the drag mechanism.

The dipole-dipole interaction energy per exciton of a gas of perfectly aligned dipolar excitons is given by $[8,9]$ :

$$
\varepsilon_{d d}(\vec{r})=\frac{4 \pi e^{2} z_{0}}{\epsilon} n_{X} \equiv \alpha n_{X},
$$

where $n_{X} \equiv n_{X}(\vec{r})$ is the 2-dimensional density of the dipolar excitons, $z_{0}$ is their dipole length, and $\epsilon$ is the background dielectric constant. The resulting dipole-dipole repulsion force,

$$
\mathbf{F}_{d d}=-\alpha \nabla n_{X},
$$

proportional to the density gradient, will drive the exciton expansion. The other effective force that is responsible for the diffusion of an exciton gas is

$$
\mathbf{F}_{D}=-\nabla \zeta
$$

Here $\zeta=k T_{X} \ln \left(1-e^{-T_{0} / T_{X}}\right)$ is the chemical potential in the non-interacting limit [8], where $T_{0}=\frac{\pi \hbar^{2} n_{X}}{2 k m_{X}}$ is the degeneracy temperature and $T_{X}$ and $m_{X}$ are the exciton gas temperature and exciton effective mass, respectively. The overall internal force driving the excitons is therefore $\mathbf{F}=\mathbf{F}_{d d}+\mathbf{F}_{D}$. The resulting exciton current $\mathbf{J}=n_{X} \mu \mathbf{F}=n_{X} \mu\left(\mathbf{F}_{d d}+\mathbf{F}_{D}\right)$ consists 
of contributions from both the dipole-dipole repulsion and the diffusion:

$$
\begin{aligned}
\mathbf{J}_{d} & =-n_{X} \mu \alpha \nabla n_{X} \\
\mathbf{J}_{D} & =-n_{X} \mu \nabla \zeta=-\mu \frac{k T_{0}}{e^{T_{0} / T_{X}}-1} \nabla n_{X}
\end{aligned}
$$

Here, $\mu$ is the exciton mobility which is, in general, a function of $|\mathbf{F}| \equiv F$.

We now construct a simplified model to obtain the dependence of $\mu$ on $F$ following the observation that interface roughness scattering is the dominant relaxation mechanism. We model the interface roughness scattering by a random distribution of quasi-elastic, hard sphere scattering centers with a characteristic mean free path $l$. In the low-field limit, the mobility, $\mu_{l f}$, is independent of $F$. In that limit, $\mu_{l f}$ is related to the diffusion coefficient through the modified Einstein relation [8] $D=\mu k T_{X} /\left(e^{T_{0} / T_{X}}-1\right)$, which in the non-degenerate limit reduces to the well-known $\mu_{l f}=D_{X} / k T_{X}$. In this picture, $D_{X}=l^{2} / \tau=l v_{t h}$, where $\tau$ is the exciton scattering time and $v_{t h}$ is the thermal velocity $v_{t h}=\sqrt{2 k T_{X} / m_{X}}$. In the high field limit however, the drift velocity $v_{d}$ depends on the force $F$ and is determined by the average velocity the exciton gains between successive collisions, given by $v_{d}=\sqrt{F l /\left(2 m_{X}\right)}$. The resulting high field limit for the mobility is then $\mu_{h f}=v_{d} / F=\sqrt{l /\left(2 m_{X} F\right)}$. We thus construct a smooth function for the mobility as a function of $F$ that conforms to the above two limits:

$$
\mu(F)=\sqrt{\frac{l /\left(2 m_{X}\right)}{F+k T_{X} / 4}} .
$$

The crossover between the two transport regimes occurs at an $F$ which corresponds to $v_{t h} \approx 4 v_{d}$. The dependence of the mobility on $v_{d} / v_{t h}$ is plotted in Fig. 19. The free exciton gas dynamics is now described by a nonlinear diffusion-drift equation,

$$
\frac{\partial n_{X}}{\partial t}+\nabla \cdot\left(\mathbf{J}_{D}+\mathbf{J}_{d}\right)+\frac{n_{X}}{\tau_{X}}-I_{X}(r, t)=0
$$

where $I_{X}(r, t)$ is the exciton source and $\tau_{X}$ is the exciton recombination lifetime. This nonlinear equation can be solved numerically.

Since $\mu$ depends on the internal force $F$, the exciton transport is in general nonlinear, analogous to nonlinear I-V characteristics of charged particles. In the case that $v_{d} \ll v_{t h}$, $\mu$ becomes independent of $F$ and the exciton transport becomes linear. However, even in the linear transport limit, the exciton expansion can be very fast, strongly driven by the dipole repulsion which can dominate over diffusion (it turns out that this is the case in the 
experiments presented in Ref. [3]). Thus, we define the following quantity to describe the importance of the fast dipole driven expansion:

$$
\gamma(r, t)=J_{d} / J_{D}=\frac{n_{X} \alpha\left(e^{T_{0} / T_{X}}-1\right)}{k T_{0}},
$$

which, in the dilute limit, reduces to just the ratio between the dipole-dipole energy term and the thermal energy of the excitons, $\gamma=\frac{\alpha n_{X}}{k T_{X}}$. When $\gamma \gg 1$, the expansion is driven by the repulsive dipole-dipole force. When $\gamma \ll 1$, the expansion becomes diffusive. An exciton gas will exhibit a fast driven expansion if its initial density profile, $n_{X}^{0} \equiv n_{X}(r=0, t=0)$, satisfies $\gamma(r=0, t=0)>1$. As a dense exciton gas starts to rapidly expand due the dipole forces, its density will decrease and the expansion will slow down. Wherever the density decreases such that $\gamma(r=0, t=0)<1$, the expansion will become diffusive, until eventually the diffusion will dominate the dynamics of the whole exciton gas.

We start our discussions by comparing the model calculations to the experimental results of Ref. 3]. There, DQW structures were used with different well widths: 80A, 100A, 120A, and $140 \mathrm{~A}$ and the corresponding $D_{X}$ were found to be (in units of $\mathrm{cm}^{2} / \mathrm{s}$ ): $0.24,0.74,2.08$, and 9.4. These numbers yield a corresponding mean free path of $l(\mathrm{~nm})=1.4,4.5,12.5,56.6$, for the experimental temperature, $T=2 K$, and for an exciton effective mass $m_{X}=0.2 m_{e}$. Note that for the wide DQW structures $l>a_{X}$ (where $a_{X} \approx 14 n m$ is the exciton Bohr radius) while for the narrow DQW's, $l<a_{X}$. Using the parameters of Ref. 3], we find that exciton drift velocity $v_{d}$ is always smaller than $v_{t h}$ even for samples with the highest $D_{X}$. The exciton transport is therefore linear with $\mu \simeq \mu_{l f}$. However, we estimate that $\gamma(r=0, t=0) \simeq 800$, indicating that the exciton cloud should exhibits an initial fast driven expansion due to the dipole pressure, and later a diffusive behavior.

For our calculations, we assume radial symmetry and an initial gaussian distribution of optically excited dipolar excitons, with a half width $\sigma_{0}=15 \mu \mathrm{m}$ and an $n_{X}^{0}=10^{11} \mathrm{~cm}^{-2}$ (unless stated otherwise), similar to the experimental conditions of Ref. [3]. We also use an exciton dipole length of $z_{0}=12 \mathrm{~nm}$. A temperature of $3 \mathrm{~K}$ and an exciton lifetime of $3 \mu \mathrm{s}$ are assumed. Fig. 1b presents calculated exciton density profiles for several different times and for $D_{X}=9.4 \mathrm{~cm}^{2} / \mathrm{s}$, showing the exciton expansion.

Fig. 2 a plots the calculated squares of the half-width - half-maximum $\left(\sigma^{2}\right)$ of the exciton density profiles at various times, for various $D_{X}$ extracted from the experiments of Ref. [3] . Both the fast initial expansion and the later slow diffusive regime are clearly seen. The 
larger the $D_{X}$, the faster the initial expansion, as $\mu$ and $D_{X}$ are linked through the scattering length. While we did not try to do any "fine tuning" of parameter fitting, there is a very good agreement with the experimental results (Fig. 4 in Ref. 3]). The only parameters for

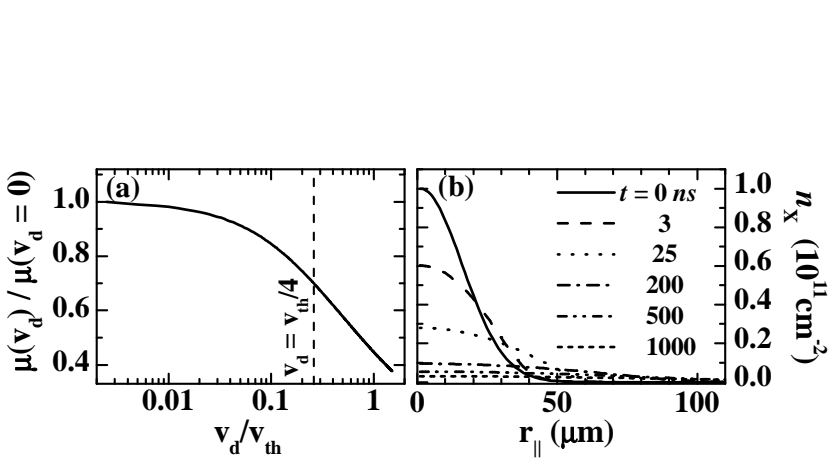

FIG. 1: (a) Normalized dipolar exciton mobility as a function of the ratio of the drift velocity to the thermal velocity $v_{d} / v_{t h}$. (b) Dipolar exciton radial density distribution profiles for different times.

The experimental data, however, seem to have a sharper transition from the driven regime to the diffusive one than the calculation. This is seen in the sharper bend of $\sigma^{2}$ with time shown in Ref. [3]. This discrepancy may be due to the heating of the exciton gas as it expands, transferring the potential energy into thermal energy by the elastic scattering processes. Such heating process is not included in our model.

Fig. $2 \mathrm{~b}$ presents the calculated time traces of the emission from different fixed radial positions of the expanding exciton gas (for $D_{X}=0.74 \mathrm{~cm}^{2} / \mathrm{s}$ ). These calculated time traces are also in a good agreement with the experiments (Fig. 2 in Ref. [3]). It seems then that this model can quite accurately approximate real experimental observations, based on the few simple assumptions given above.

After establishing the validity of this picture, we are now ready to discuss some of its important consequences. It is already clear that a dense and mobile dipolar gas will tend to expand quickly due to the internal repulsive forces. If the initial cloud is small, the expansion will quickly reduce the exciton gas density and will limit the time the high density condition can be sustained. We start by discussing small initial exciton clouds, excited by tightly focused laser beams, which is the most common experimental approach. In Fig. 3 a we plot 


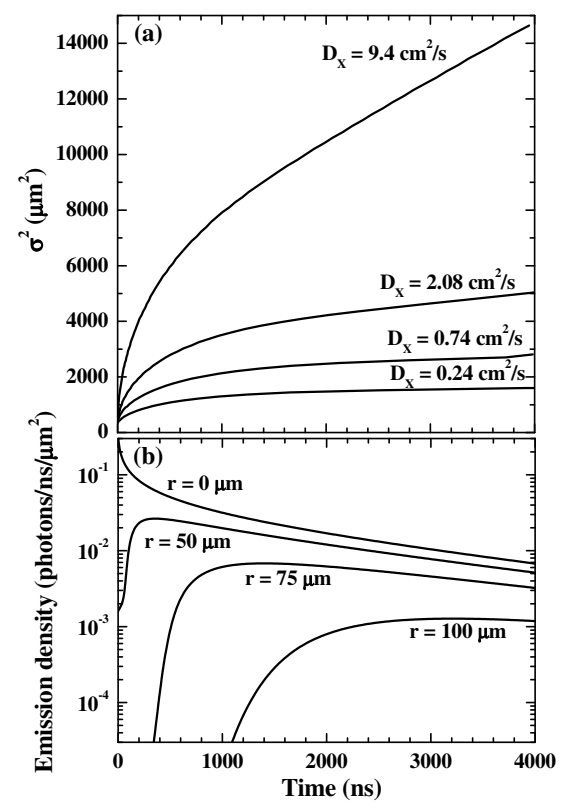

FIG. 2: (a) $\sigma^{2}$ of the density distribution profiles as a function of time, for the diffusion coefficients of Ref. 3]. (b) Calculated dipolar exciton emission density as a function of time from different radial positions of the exciton cloud, corresponding to the experimental positions measured in Ref. 3]. The parameters for the calculations in both (a) and (b) are given in the text.

the central density of an expanding, initially small $(\sigma=15 \mu \mathrm{m})$, high mobility exciton gas $\left(D_{X}=10 \mathrm{~cm}^{2} / \mathrm{s}\right)$. The calculations are performed with a gas temperature of $T_{X}=2 \mathrm{~K}$, as a function of time, for different initial exciton densities $n_{X}^{0}$. the horizontal dash-dotted line represents the density, $n_{X}^{c}$, in which the exciton thermal de-Broglie wavelength is equal to the exciton inter-particle distance, i.e., $\lambda_{d B}^{2} n_{X}^{c} / g_{X}=1$. One expects to observe quantum statistical effects due to Bose-Einstein distribution only for densities larger than $n_{X}^{c}$. As can be seen, within less than 30ns, the density drops below $n_{X}^{c}$ even for high initial gas densities. This time is much shorter than the exciton lifetime of $3 \mu \mathrm{s}$. Again, this is a consequence of the fast initial expansion. Fig. 3b shows the similar density-time traces for an initial density $n_{X}^{0}=10^{11} \mathrm{~cm}^{-2}$ for different temperatures. The circles on each density-time trace mark $n_{X}^{c}$ for the particular temperature, given by: $n_{X}^{c}=m_{X} g k T_{X} /\left(2 \pi \hbar^{2}\right)$, and the curved dashed-dot line is a guide to the eye. While decreasing temperature results in an increased mobility for a given $D_{X}$, there is also a linear decrease in $n_{X}^{c}$, resulting in an improvement of the time interval in which $n_{X}>n_{X}^{c}$. However, even at $T_{X}=0.5 \mathrm{~K}$ this time is still under $70 \mathrm{~ns}$, 
significantly shorter than the exciton radiative lifetime, $\tau_{X}$. Fig. 35 shows similar traces for different diffusion coefficients. A smaller diffusion coefficient is related to a smaller mean free path $l$, which in turn yields less expansion and less density decrease.

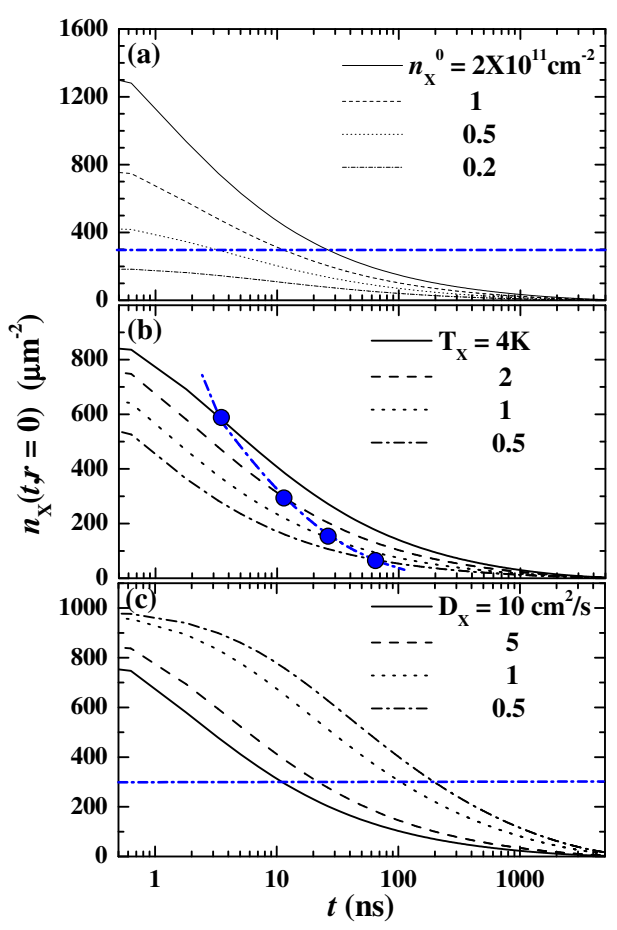

FIG. 3: Center exciton densities as a function of time, $n_{X}(t, r=0)$ for (a) different initial center densities $n_{X}^{0}$, (b) different exciton gas temperatures $T_{X}$, and (c) Different exciton diffusion coefficients $D_{X}$. The relevant parameters for the calculations in (a), (b) and (c) are given in the text. The dashed-dot lines in (a) and (c) and the circles in (b) mark the de-Broglie density $n_{X}^{c}$.

Fig. 4a,b summarize the above results. The time interval $\Delta t$ in which $n_{X}>n_{X}^{c}$ as a function of the exciton gas temperature, for various $D_{X}$ values is shown in Fig. 4a and for various initial densities $n_{X}^{0}$ in Fig. 4b. For high mobility (diffusivity) samples, decreasing the temperature and increasing the initial density only slightly increases $\Delta t$, to values still much smaller than $\tau_{X}$. This is due to the fast driven expansion. For low mobility samples, at very low temperatures, $\Delta t$ can be in the microsecond range, comparable to $\tau_{X}$. For these samples, however, the mean free path, $l$, is much smaller than the exciton Bohr radius, $a_{X}$. This may complicate the observation of Bose-Einstein quantum statistical effects in general and excitonic BEC in particular due to the disorder induced localization of the excitons. 


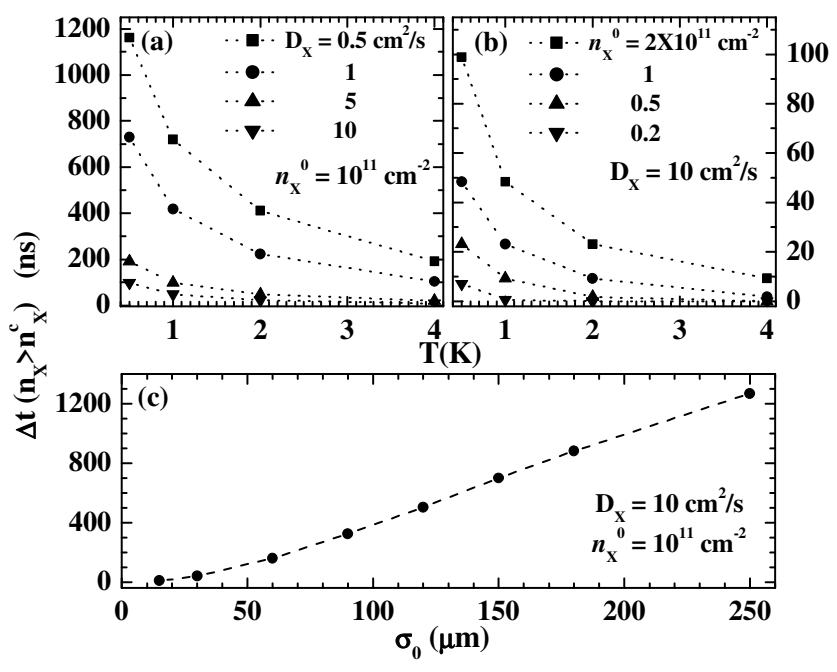

FIG. 4: Time interval $\Delta t$ in which the center dipolar exciton gas density, $n_{X}(r=0)$ is larger than the de-Broglie density, $n_{X}^{c}$, as a function of the exciton gas temperature, $T_{X}$. (a) shows the results for different diffusion coefficients, $D_{X}$ while (b) shows different initial center densities, $n_{X}^{0}$. (c) shows $\Delta t$ as a function of the initial size of the exciton cloud for $T_{X}=2 K$.

Fig. 4t shows the dependence of $\Delta t$ on the initial size of the exciton cloud or $D_{X}=$ $10 \mathrm{~cm}^{2} / \mathrm{s}$ and $T_{X}=2 K$. As the initial size increases (keeping the same center density), the dipole driving force decreases $F_{d d} \propto\left|\nabla n_{X}\right| \propto 1 / \sigma_{0}$, and so does the expansion rate. Starting with large enough initial exciton clouds leads to a much smaller expansion rates and thus to $\Delta t$ values comparable to the exciton lifetime $\tau_{X}$.

To conclude this analysis, there are two strategies to keep a high mobility dipolar exciton gas dense enough for long enough time intervals. The first is to optically excite an initially very large cloud, thus suppressing the fast expansion. This approach, however, is not favorable since it involves high excitation power which could lead to complications such as increased heating of the sample. The other strategy is to design an artificial spatial confinement in the QW plane. For such dipolar excitons, the trapping potential can be induced either through a local strain in the QW [10] or by locally applying the external electrostatic potential[11]. These exciton traps not only keep the excitons dense, but also eliminate the extra heating (due to the dipole-dipole potential) of a free-expanding exciton gas mentioned previously and breaking the 2D translational which allows for the the formation of BEC.

It is interesting to compare the two characteristic densities of this problem. The first is 
the de-Broglie density, $n_{X}^{c}$, that marks the onset of a distinguishable Bose-Einstein statistics effects. The second density is the critical density for the driven expansion regime, $n_{d}^{c}=$ $k T_{X} / \alpha$, derived by setting $\gamma=1$ in the linear transport limit and assuming non-degenerate gas. $n_{d}^{c}$ marks the transition from a fast dipole-pressure driven exciton expansion to a diffusive expansion. Their ratio yields,

$$
\frac{n_{X}^{c}}{n_{d}^{c}}=\frac{2 m_{X} g e^{2} z_{0}}{\hbar \epsilon} \approx 20,
$$

for typical GaAs DQW structures and is independent of temperature, justifying the above assumption. This means that a degenerate Bose-Einstein gas of dipolar excitons is always strongly driven by the dipolar pressure. This can have some interesting consequences. For example, it indicates that a highly degenerate dipolar gas will expand quickly, in contrast to most atomic BEC gases that tend to expand very slowly. Also, it is not clear how a driven expansion of a highly degenerate exciton gas would differ from that of a classical gas and how would the scattering of such an exciton gas from well width fluctuations would differ from the simplistic, ballistic "billiard ball" scattering model we have used. Our model also suggests that for high mobility samples, such as the ones used in Ref. [3], the mobility is on the edge of the nonlinear transport regime. By cooling the exciton gas further or by growing wider samples the predicted range for the study of highly nonlinear transport due to the internal dipole pressure can be reached for the first time.

We thank Loren Pfeiffer, P.M. Platzman, David Snoke and Xing Wei for enlightening discussions.

[1] D. Snoke, Science 298, 1368 (2002).

[2] L. V. Butov, J. Phys.: Condens. Matter. 16 R1577 (2004).

[3] Z. Vörös, R. Balili, D.W. Snoke, L. N. Pfeiffer, and K. West, Phys. Rev. Lett. 94, 226401 (2005), cond-mat/0504151 (2005).

[4] H. Sakaki, T. Noda, K. Hirakawa, M. Tanaka, and T. Matsusue, Appl. Phys. Lett. 51, 1934 (1987).

[5] R.E. Prange and T. W. Nee, Phys. Rev. 168, 779 (1968).

[6] A. Gold, Solid State Commun. 60, 531 (1986).

[7] A. Gold, Phy. Rev. B 35, 723 (1987). 
[8] A. Ivanov, P.B. Littlewood and H. Haug, Phys. Rev. B 59, 5032 (1999); A. Ivanov, Europhys. Lett. 59(4), 586 (2002).

[9] S. Ben-Tabou de-Leon and B. Laikhtman, Phys. Rev. B 63, 125306 (2001).

[10] D. Snoke, Y. Liu, Z. Voros, L. N. Pfeiffer, and K. West, Solid State Commun. 134, 37 (2005).

[11] R. Rapaport, G. Chen, S. Simon, O. Mitrofanov, L. N. Pfeiffer, and P. M. Platzman, Phys. Rev. B, 72, 000000 (2005), cond-mat/0504178, (2005). 